\title{
Influence of Co Underlayer Thickness on Mass Resolving Power in Field Evaporated Cu/Co Bilayer
}

\author{
K. Tippey, B.C. Hornbuckle, B. Fu, and G.B. Thompson \\ University of Alabama, Department of Metallurgical \& Materials Engineering, Tuscaloosa, AL \\ 35487-0202, USA
}

A critical aspect of atom probe tomography is the ability to correctly range the time of flight mass spectrum. If the field evaporation is not optimized where atoms field evaporate at times not at the peak field conditions, the mass spectrum will yield long tails. These mass-to-charge tails are concerning in that they can lead to peak overlaps between different species as well as incorrect compositional determination if not all the ions are ranged and counted correctly.

In the current study, the influence of a Co underlayer on the mass resolving power of $\mathrm{Cu}$ is studied. $\mathrm{Co} / \mathrm{Cu}$ based multilayers have received interest because of their use in giant magnetoresistance devices. The bilayer studied in this work was sputter-deposited on a n-doped Si pillar with a $5 \mathrm{~nm}$ Cr layer to improve the adhesion of the bilayer to the substrate. A $200 \mathrm{~nm}$ $\mathrm{Cu}$ film, with no Co, was deposited to serve as a base line comparison to a $80 \mathrm{~nm} \mathrm{Co} / 200 \mathrm{~nm} \mathrm{Cu}$ and a $160 \mathrm{~nm} \mathrm{Co} / 200 \mathrm{~nm} \mathrm{Cu}$ bilayer. The Si post was a 2 x $2 \mu \mathrm{m}$ flat surface requiring annular ion milling using a focus ion beam to yield the correct needle-shape geometry required field evaporation. An in situ $2 \mu \mathrm{m}$ Pt coating was deposited over the film to provide a protective surface during the shaping of the needle. Ideally, the milling was ceased at the $\mathrm{Pt} / \mathrm{Cu}$ interface. Particular care was done to ensure that the taper angle and radius of curvature was equivalent between each specimen. A representative tip is shown in figure 1. Since experimental variation will occur, three specimens for each Co thickness - 0, 80, and $160 \mathrm{~nm}$ - were made. The time-offlight mass spectrums were averaged to eliminate small variations that could be present between tip geometry as well as in laser focus point fluctuations on the tips during field evaporation. Evaporation was continued from the $\mathrm{Cu}$ until it penetrated $\sim 50 \mathrm{~nm}$ into either the Co layer or $\mathrm{Si}$ substrate (if no Co underlayer was not present).

Each film was run in a Cameca Local Electrode Atom Probe (LEAP) 3000XSi. The tips were held at a base temperature of $30 \mathrm{~K}$, laser pulsed with a $\lambda=532 \mathrm{~nm}$ and at an energy density of 33 $\mathrm{pJ} / \mathrm{mm}^{2}$ at $250 \mathrm{kHz}$ (one experiment was done at $10 \mathrm{kHz}$ ). Targeted evaporation was set a $0.5 \%$. During field evaporation, the standing voltage ranged from $\sim 2 \mathrm{keV}$ to $\sim 5 \mathrm{keV}$ as the tip evolved.

As the Co thickness increased, the full width half maximum (FWHM) increased with increasing $\mathrm{Co}$ thickness, as seen in figures 2 and plotted in figure 3 . The thermal conductivities for $\mathrm{Cu}$ and Co is $\sim 75 \mathrm{~W} / \mathrm{m}^{*} \mathrm{~K}$ and $10 \mathrm{~W} / \mathrm{m}^{*} \mathrm{~K}$, respectively. The lower thermal conductivity of Co can cause a retention of heat within the specimen tip which is manifested by the uncontrolled field evaporation of $\mathrm{Cu}$ after the pulse ceases. Interestingly, in prior work of atom probe tomography in $\mathrm{Cu} / \mathrm{Co}$ multilayers [1], this mass resolving degradation was not noted. This maybe a result that these previous studies involved smaller volumes as a consequence of an individual films being one to a few nanometers thick.

The mechanism of field evaporation, wither an electro-optical or thermal effect, by pulsed laser has been a matter of discussion [2]. To determine if the degradation of Cu's FWHM is dominated by electro-optical or thermal effects, the pulse frequency of the laser was reduced from $250 \mathrm{kHz}$ to $10 \mathrm{kHz}$ keeping all other atom probe run conditions equivalent for a $160 \mathrm{~nm}$ $\mathrm{Co} / 200 \mathrm{~nm} \mathrm{Cu}$ bilayer. As can be seen in figure 3, a significant improvement was achieved. By reducing the pulse frequency, the specimen tip was provided adequate time for the heat to be 
extracted from the Co layer prior to the next pulse. If it was dominated by an electro-optical mechanism, wither pulsed at $250 \mathrm{kHz}$ or $10 \mathrm{kHz}$, the mechanism would be the same and the mass spectrums would be equivalent. Based on the improved FWHM with decreasing laser pulse frequency, the dominate mechanism for field evaporation appears to be primarily or solely a thermal assisted effect.

References:

[1] A.K. Petford-Long et al. Microscopy and Microanalysis 10(3), pp. 366-372 (2004)

[2] B. Gault et al. Ultramicroscopy 107(9), pp. 713-719 (2007)

[3] This work was funded under NSF-DMR-1207220

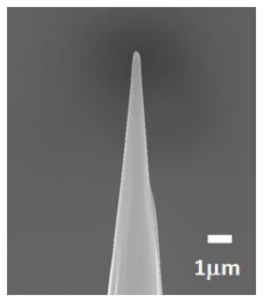

Figure 1: SEM micrograph showing a representative ion milled atom probe tip with the bilayer.
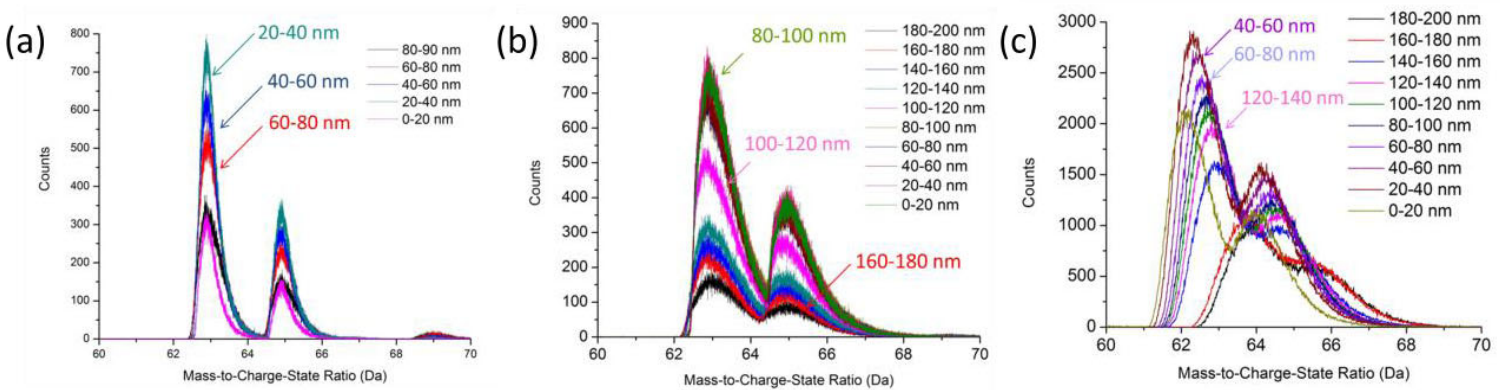

Figure 2: Cu's mass spectrums for the following Co underlayer thicknesses: (a) $0 \mathrm{~nm}$ (b) $80 \mathrm{~nm}$ and (c) $160 \mathrm{~nm}$. The sub-sectioned 20 thicknesses represent the data volumes used for the FWHM analysis referenced to the distance from either the Si-Cu interface for (a) or from the Co$\mathrm{Cu}$ interface for (b) and (c).

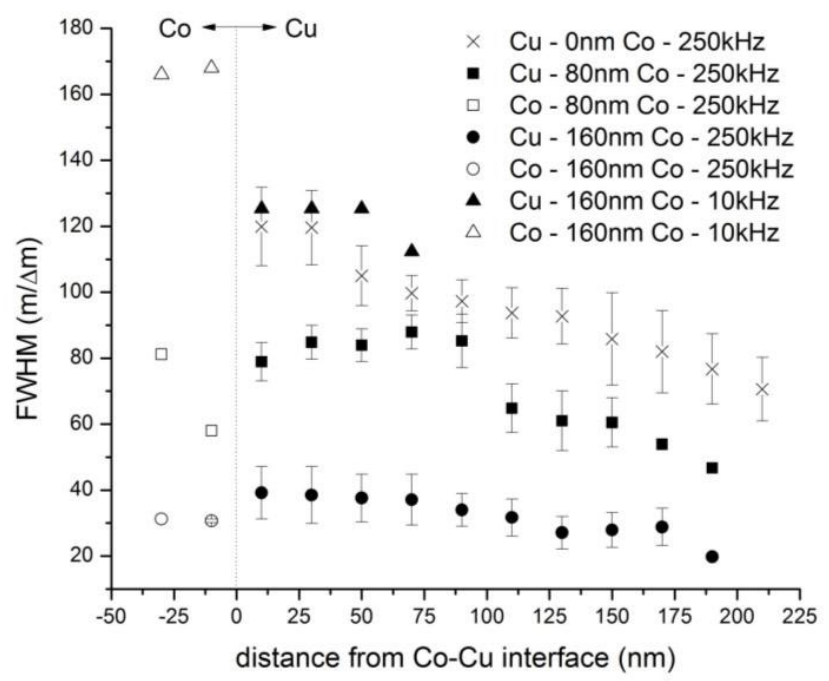

Figure 3: Plot of FWHM vs. distance from the $\mathrm{Co}-\mathrm{Cu}$ interface. 